\title{
The mean severity score and its correlation with common computed tomography chest manifestations in Egyptian patients with COVID-2019 pneumonia
}

Mona A. F. Hafezid

\begin{abstract}
Background: Computed tomography $(\mathrm{CT})$ is one of the main diagnostic tools for early detection and management of coronavirus disease 2019 (COVID-19) pneumonia. This study aims to highlight the commonly encountered CT findings in patients with COVID-19 pneumonia in Egypt and the mean severity score and its correlation with the imaging findings. This study involved 200 patients with pathologically confirmed COVID-19 infection; non-contrast $\mathrm{CT}$ chest was performed for all cases; in addition, CT findings and severity score (CT-SS) were then assessed using descriptive analysis, and the correlation between the $C T$ findings and disease severity was assessed.

Results: The ground-glass densities and peripheral adhesions were the most typical $C T$ findings. Prominent interlobular septations; bronchial thickening/dilatation; CT signs of crazy-paving, halo, and reversed halo; and reactive mediastinal lymphadenopathy were significantly correlated with disease severity. The mean CT-SS of Egyptian patients with COVID-19 pneumonia was 11.2 (mild to moderate severity).

Conclusion: Multislice CT played a vital role in the early identification of Egyptian patients with COVID-19 pneumonia. The assessment of the CT severity score of COVID-19 is essential for the extent of pneumonia involvement to help clinicians achieve the purpose of early diagnosis and accurate treatment.
\end{abstract}

Keywords: Computed tomography, COVID-19 pneumonia, Chest, Egyptian

\section{Background}

Coronavirus disease 2019 (COVID-19) pneumonia is a recently discovered rapidly spreading acute respiratory syndrome [1]. Egypt is one of the top 40 countries worldwide according to the total number of cases in $\mathrm{Au}$ gust 2020 [2].

During the current epidemic in Egypt, computed tomography $(\mathrm{CT})$ is used as the main diagnostic tool for early detection and management of COVID-19 pneumonia. Laboratory tests were the standard for diagnosing COVID-19 pneumonia. However, sometimes, they are

\section{Correspondence: mona.fouad@kasralainy.edu.eg;}

Mona.fouad@kasralainy.edu.eg

Radiology and Intervention Department, Kasralainy Hospital, Cairo University School of Medicine, Kasr Al-Aini Street, Cairo 11562, Egypt unavailable in an emergency, and the results are unavailable immediately [3, 4].

Chest CT has a $97 \%$ sensitivity for the diagnosis of COVID-19 pneumonia after a mean interval of 5 days [3]. The typical chest CT findings in COVID-19 pneumonia are bilateral, peripheral, and basal predominant ground-glass opacities (GGOs) with or without consolidation and bronchovascular thickening [5]. In addition, atypical chest $\mathrm{CT}$ findings include central upper lobe predominance, masses, nodules, cavitations, tree-in-bud sign, lymphadenopathy, and pleural effusion [6].

Quantitative and semiquantitative indicators that evaluate the chest CT severity score (CT-SS) of lung inflammation in COVID-19 assess COVID-19 burden and provide an objective approach in rapidly identifying 
patients in need of hospital admission. The CT-SS is an adjustment of a method previously used in patients with severe acute respiratory syndrome (SARS) to describe the extent of the disease in the lungs, which was correlated with clinical and laboratory parameters [7].

This study highlights the commonly encountered CT findings in patients with COVID-19 pneumonia in Egypt and the mean CT-SS and its correlation with the imaging findings.

\section{Methods}

In this cross-sectional prospective study, 200 patients including 111 males (55.5\%) and 89 females (44.5\%) were enrolled in the period from June 30, 2020, to August 30, 2020. The male-to-female distribution was 1.2:1.

All patients clinically diagnosed or suspected with COVID-19 underwent high-resolution CT/non-contrastenhanced multi-slice chest CT. Patients with chest CT findings suspicious of COVID-19 pneumonia were included in the study (COVID-19 Reporting and Data System [CO-RADS] 4, 5, and 6). However, normal CT chest and those with any other chronic CT chest disease as of pulmonary edema and interstitial lung disease (CORADS 1,2, or 3) were excluded from the study.

All patients were subjected to full clinical data taking including age, sex, exposure history, clinical complaint, and laboratory parameters.

PCR laboratory tests were performed for all patients before or after chest CT.

All patients underwent non-contrast-enhanced chest $C T$ in the radiology department using a Siemens 16channel scope (CTAWP92544; Siemens Healthineers, Erlangen, Germany). All volumetric chest CT were assessed at lung window of $1500 \mathrm{WW}$ and $-500 \mathrm{WL}$ and mediastinal window of $400 \mathrm{WW}$ and $60 \mathrm{WL}$ using $2 \mathrm{D}$ coronal and sagittal planes for better assessment of the extent of the disease.

A radiologist with 15 years of experience in thoracic imaging performed the CT image analysis; the following parameters in each $\mathrm{CT}$ were assessed.

\section{Location and distribution of disease}

The location and distribution of the disease were unilateral or bilateral; peripheral, central, or both; and upper lobe predominance, lower lobe predominance, or both.

\section{$\mathrm{CT}$ chest findings}

The CT chest findings were the presence of groundglass opacification; consolidation; special CT chest signs such as crazy-paving, halo, and reversed halo signs; spiderweb appearance; subpleural sparing; interlobular septal thickening; intralobular septal thickening; parenchymal band; subpleural band; cyst; nodule; vascular thickening; bronchial thickening; pleural thickening; pleural reaction; pleural effusion; and reactive lymph nodes (exceeding $1 \mathrm{~cm}$ in short-axis diameter).

\section{CO-RADS based on the CT findings}

The level of suspicion of COVID-19 infection is graded: CORADS score [8]-CO-RADS 1: COVID-19 is highly unlikely, $\mathrm{CT}$ is normal, or there are findings indicating a noninfectious disease; CO-RADS 2: the level of suspicion of COVID-19 infection is low, and CT findings are consistent with other infections; CO-RADS 3: COVID-19 infection is unsure or indeterminate, and $\mathrm{CT}$ abnormalities indicate infection but are unsure whether COVID-19 is involved; CORADS 4: the level of suspicion is high, and most CT findings are suspicious but not extremely typical as unilateral groundglass, confluent, or multifocal consolidations without a typical location or any other typical findings; and CO-RADS 5: the level of suspicion is high with typical CT findings.

\section{Semiquantitative scoring system}

A semiquantitative scoring system was used to quantitatively estimate the pulmonary involvement of all these abnormalities based on the area involved. The CT-SS was calculated based on the extent of lobar involvement. Each of the five lung lobes was visually scored on a scale of $0-5$, with 0 indicating no involvement, 1 indicating less than 5\% involvement, 2 indicating 5-25\% involvement, 3 indicating $26-49 \%$ involvement, 4 indicating $50-75 \%$ involvement, and 5 indicating more than $75 \%$ involvement. The total CT score was the sum of the individual lobar scores and ranged from 0 (no involvement) to 25 (maximum involvement) $[9,10]$.

\section{Statistical analysis}

The Statistical Package for the Social Sciences (version 24; IBM Corp., Armonk, NY, USA) was used in the data manipulation and significance testing. Categorical data were expressed as numbers and percentages, whereas numerical data were summarized as medians. The patients were stratified into two groups according to their CT-SS: those with CT-SS of 1-17 were considered mild, whereas those with CT-SS of $18-25$ were considered severe. The chi-square correlation analysis was conducted, and $p$ values of $<0.1$ were used to denote statistical significance. The research protocol was approved by the Institutional Human Research Ethics Committee and conducted in accordance with the Declaration of Helsinki. A written informed consent was taken from all patients who participated in the study.

\section{Results}

Demographic, severity, and clinical characteristics

This study involved 200 patients, including 111 males (55.5\%) and 89 females (44.5\%), with their ages ranging from 20 to 87 years (average age, 52.6 years). 
The mild group (CT-SS of 1-17) consisted of 180 patients, whereas the severe group (CT-SS of 18-25) was composed of 20 patients.

The most affected age group was the 51-75-year age group (99 patients; $49.5 \%$ ) followed by the $26-50$-year age group (90 patients; 45\%), then the < 25-year age group (six patients; $3 \%$ ), and the $>75$-year age group (five patients; $2.5 \%$ ). Disease severity is significantly correlated with the 51-75 year age group ( $p=0.033)$.

The most presenting symptoms are displayed in Table 1. Disease severity was significantly correlated with lower respiratory symptoms, in particular, cough, and the presence of chronic diseases.

\section{CT imaging findings}

Chest CT findings were assessed and analyzed for all examined patients.

Accordingly, the disease distribution was assessed; most of the cases presented with bilateral involvement with peripheral and lower lobe predominance (Table 2 and Fig. 1). Both peripheral and central distributions showed significance with disease severity.

According to the CT findings, the most prominent features are GGO with vascular pleural thickening and interlobular septations, and parenchymal and subpleural bands were the most constant imaging features (Fig. 2).

A significant correlation was found between disease severity and consolidation, interlobular septation, bronchial thickening/dilatation, pulmonary nodule, and lymphadenopathy.

Among several specific CT signs noted with COVID19 pneumonia, the crazy-paving sign was the most encountered CT sign followed by the subpleural sparing sign. A significant correlation was found between disease severity and crazy-paving, halo, and reversed halo signs (Table 3 and Figs. 3, 4, and 5).

Reactive lymphadenopathy exceeding $1 \mathrm{~cm}$ in shortaxis diameter affected the paratracheal, retrocaval, and prevascular groups at levels $3 \mathrm{~A}$ and 6 the most, followed by the level 5 lymph node group. The cortical estimated density ranged from $23 \mathrm{HU}$ to $141 \mathrm{HU}$, with an average density of $91.8 \mathrm{HU}$ (Fig. 6).

According to the CO-RADS classification, one patient was classified as CO-RADS 6 (known as COVID-19 pneumonia), 45 patients showed CO-RADS 4 findings, and 154 patients were classified as CO-RADS 5.

The CT-SS were from 1 to 24 , with a mean value of 11.2 and a median value of 13 . Among all patients, $40.5 \%$ had CT-SS of 11-15 (Table 4).

\section{Discussion}

COVID-19 is a highly infectious disease that has been spreading widely worldwide. Early diagnosis is an essential disease management strategy [11]. However, insufficient laboratory kits caused a challenge and dramatic dissemination of the disease [12]. Therefore, radiology, such as X-ray and CT, had become the principal method for diagnosis during the COVID-19 outbreak.

Chest CT could be an important complement for disease diagnosis as it assesses the extent and severity of the disease, which could express the disease burden [7]. Chest CT has a high sensitivity and a low specificity. Due to this low specificity, chest CT could hardly distinguish COVID-19 pneumonia from other diseases, such as community-acquired pneumonia and other noninfectious causes of acute GGO $[3,13]$.

The most common clinical symptoms of patients with COVID-19 are fever, cough, dyspnea, and fatigue [7]. In this study, fever and lower respiratory symptoms are the most common presenting symptoms in $69.5 \%$ and $66 \%$ of the patients, respectively.

The CT-SS was significantly higher in the $>75$-year age group than in the 26-50-year age group ( $p=$ 0.0012). Furthermore, the CT-SS was significantly higher in the 51-75-year age group than in the 26-50-year age group $(p=0.0367)$. No statistical significance was observed in the CT-SS between the 51-75-year age group and the $>75$-year age group $(p=0.3605)$. The CT-SS could help stratify patients' risks and predict the shortterm outcomes of patients with COVID-19 pneumonia

Table 1 The common presenting symptoms, number of cases, and significance of severity with some significance in cases with no complain

\begin{tabular}{lll}
\hline Clinical complain & Number and percentages of cases & $\boldsymbol{p}$ value \\
\hline No complain & $25(12.5 \%)$ & $0.075^{*}$ \\
Upper respiratory symptom (sore throat) & $22(11 \%)$ & 0.175 \\
Lower respiratory symptoms (cough, dyspnea, tachypnea, chest pain) & $132(66 \%)$ & $0.059^{*} / 0.108 / 0.580 / 0.450$ \\
Fever & $139(69.5 \%)$ & 0.113 \\
Fatigue & $34(17 \%)$ & 0.856 \\
Gastrointestinal symptoms (abdominal pain, diarrhea) & $5(2.5 \%)$ & $0.580 / 0.501$ \\
Chronic disease & $51(25.5 \%)$ & $0.035^{*}$ \\
\hline
\end{tabular}

${ }^{*} p$ value $<0.1$ : significant 
Table 2 Number and percentages of cases according to their distribution and their significance of severity

\begin{tabular}{lll}
\hline Location & Number and percentage of cases & Significance $(\boldsymbol{p}$ value $)$ \\
\hline Unilateral & $13(6.5 \%)$ & 0.214 \\
Bilateral & $187(93.5 \%)$ & 0.214 \\
Peripheral & $179(89.5 \%)$ & $0.026^{*}$ \\
Central & $2(1 \%)$ & 0.636 \\
Both peripheral and central & $19(9.5 \%)$ & $0.013^{*}$ \\
More severity score for lower lobes & $111(55.5 \%)$ & 0.602 \\
More severity score for upper lobes & $22(11 \%)$ & 0.366 \\
Same severity score for upper and lower lobes & $66(33 \%)$ & 0.229 \\
More severity score for middle lobe/lingula & $1(0.5 \%)$ & 0.738 \\
\hline
\end{tabular}

${ }^{*} p$ value $<0.1$ : significant

[9]. Moreover, in this study, the 51-75-year age group had the highest CT-SS $(p=0.033)$.

Regarding disease distribution, all studies have indicated that COVID-19 has typical peripheral and subpleural distributions, and in most patients, COVID-19 involves multiple lobes, particularly the lower lobes [3]. In this study, $93.5 \%$ of all patients had bilateral involvement, whereas $89.5 \%$ had peripheral involvement. Higher severity scores for the lower lobe were observed in $55.5 \%$ of the patients. These results conform to those of the study by Salehi et al. [14] who reported bilateral involvement and peripheral distribution in $87.5 \%$ and
$76.0 \%$ of their patients, respectively; however, many studies such as that by Zhou et al. [15] have reported that $77.4 \%$ of the patients had predominantly peripheral distribution of lesions; the mean CT-SS for the upper zone was significantly lower than that for the middle and lower zones, and no significant difference in the mean CT-SS was observed between the middle and lower zones [9].

In this study, $13(6.5 \%)$ patients were unilateral with 11 cases $(84.6 \%)$ involving the lower lobes, one involving both the upper and lower lobes, and one involving the middle lobe. Zhou et al. [15] have stated that in the early

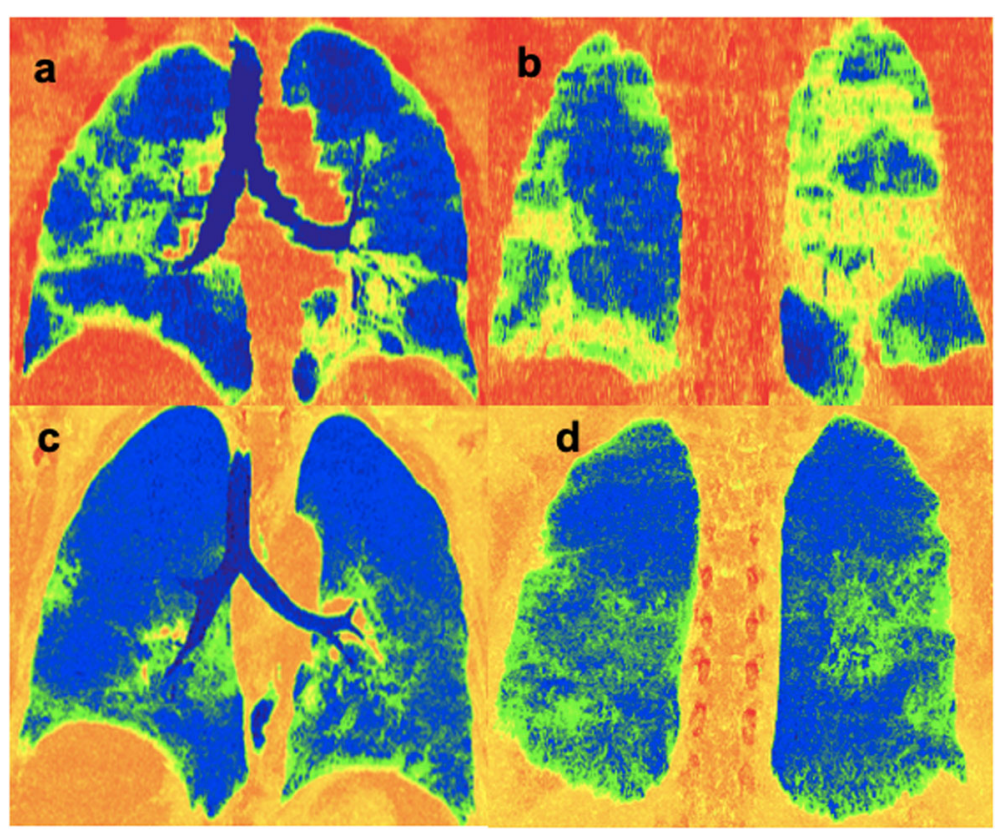

Fig. 1 Colored spectrum two-dimensional coronal lung mid- and posterior thick slap $(\mathbf{a}, \mathbf{b})$ in a 44-year-old male patient complaining of fever, dyspnea, and cough. The patient was classified as CO-RADS 5 with a CT-SS of 20. It shows the asymmetric mainly peripheral multilobar distribution of the parenchymal lesions (green and yellow colors). c, d A 68-year-old female patient complaining of fever, dyspnea, and cough. The patient was classified as CO-RADS 5 with a CT-SS of 11. It shows the asymmetric mainly peripheral lower lobe distribution of parenchymal lesions (green colour) 


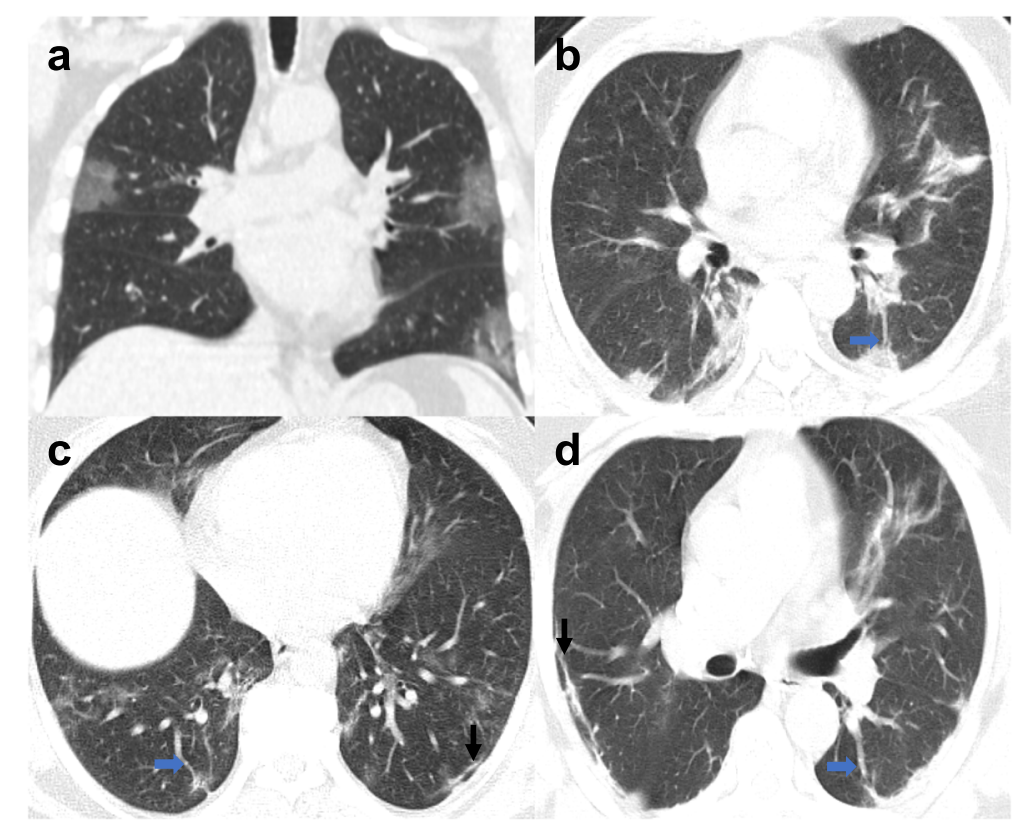

Fig. 2 Two-dimensional coronal reconstruction (a) and axial CT image lung window (b, c) in two different cases with COVID-19 pneumonia, showing the typical appearance of bilateral peripheral patchy ground-glass densities $(\mathbf{a}, \mathbf{c})$ and/or consolidations (b), subpleural band (black arrows), and vascular dilatation (blue arrows)

Table 3 CT chest findings in cases with COVID-19 pneumonia, number, and percentages of cases with a severity significance value

\begin{tabular}{lll}
\hline CT chest finding & Number and percentages of cases affected & Significance $\boldsymbol{p}$ value \\
\hline Consolidation & $60(30 \%)$ & $0.040^{*}$ \\
Ground-glass opacity (GGO) & $199(99.5 \%)$ & 0.738 \\
Inter- and intralobular septations & $144(72 \%)$ & $0.003^{*} / 0.110$ \\
Parenchymal bands & $157(78.5 \%)$ & 0.456 \\
Subpleural bands & $138(69 \%)$ & 0.919 \\
Bronchial thickening/dilatation & $118(59 \%)$ & $0.003^{*}$ \\
Vascular thickening & $185(92.5 \%)$ & 0.179 \\
Cyst & $20(10 \%)$ & 0.116 \\
Nodule & $40(20 \%)$ & $0.005^{*}$ \\
Pleural thickening & $153(76.5 \%)$ & 0.133 \\
Pleural reaction & $6(3 \%)$ & 0.407 \\
Pleural effusion & $7(3.5 \%)$ & 0.369 \\
Lymphadenopathy & $66(33 \%)$ & $0.088^{*}$ \\
Crazy-paving sign & $73(36.5 \%)$ & $0.000^{*}$ \\
Halo sign & $22(11 \%)$ & $0.097^{*}$ \\
Reversed halo sign & $24(12 \%)$ & $0.082^{*}$ \\
Spider web sign & $23(11.5 \%)$ & 0.605 \\
Subpleural sparing & $53(26.5 \%)$ & 0.873
\end{tabular}

${ }^{*} p$ value $<0.1$ : significant 


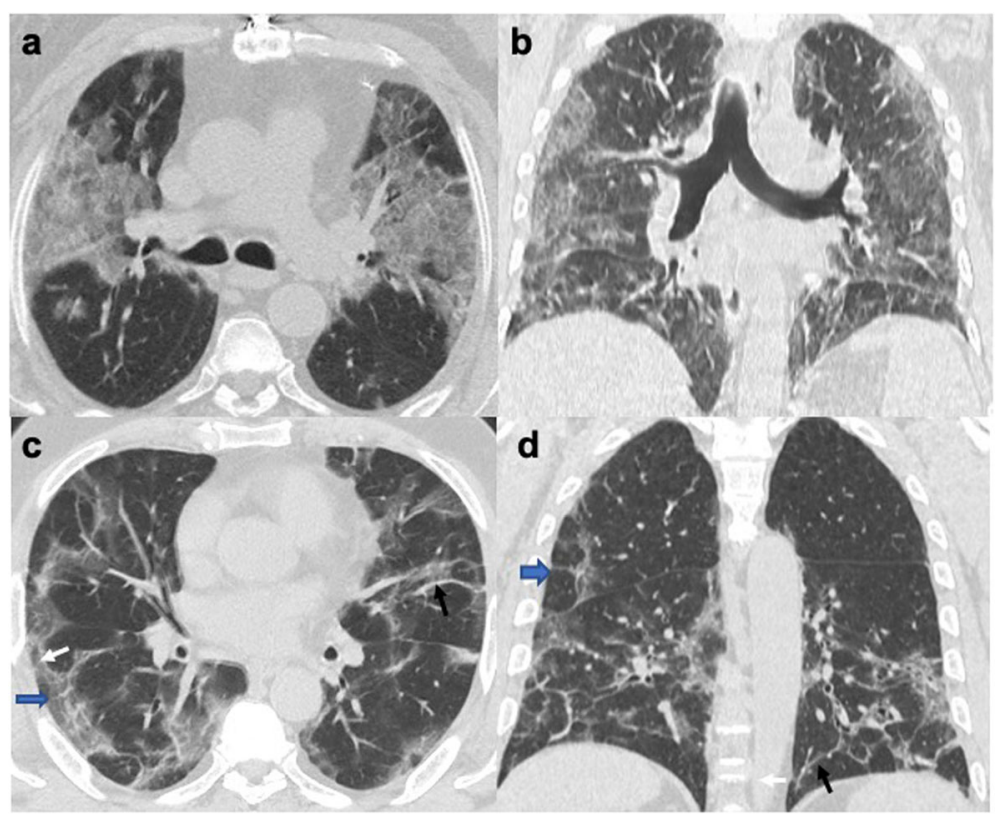

Fig. 3 Axial (a, c) and coronal (b, d) CT slices of the lung window in different cases with COVID-19 pneumonia (a, b) showing the "crazy-paving sign" shown as bilateral ground-glass density with prominent interlobular and intralobular septations. c, d The bilateral parenchymal bands (black arrows), inter- and intralobular septations (blue arrows), and subpleural bands (white arrows), forming the spiderweb sign

phase of the disease, the GGO may present as a unifocal lesion, most commonly located in the inferior lobe of the right lung.

Regarding CT chest findings, all studies have indicated that the main CT feature of COVID-19 pneumonia is the presence of multifocal bilateral patchy GGOs with or without consolidation and with interlobular septal and vascular thickening $[3,5]$. In this study, the major CT abnormalities observed were GGO in $99.5 \%$, vascular dilatation in $92.5 \%$, parenchymal bands in $78.5 \%$, interlobular septal thickening in $72 \%$, subpleural band in $69 \%$, and consolidation in $30 \%$ of the patients. These results conform to those of many studies that reported that the frequencies of different CT abnormalities were as follows: GGO was observed in $86-91 \%$ of the cases, consolidation in $39-63 \%$, fibrotic streaks in $56.5 \%$, subpleural line in $20-33.9 \%$, and interlobular septal thickening in 59\% [14-17]. However, Zhou et al. [15] have reported that $40.3 \%$ of the cases had GGO and $54.8 \%$ had microvascular dilation sign.

The characteristic sign was the "crazy-paving sign," which was observed in $36.5 \%$ of the cases, which is characterized by the reticular interlobular septa thickening within the patchy GGO, which had been reported in SARS. The "spiderweb sign" was observed in $11.5 \%$ of the patients. The "spiderweb sign" is characterized by a triangular or angular GGO under the pleura with the internal interlobular septa thickened like a net. The adjacent pleurae were pulled and formed a spiderweb-like shape in the corner. Wu et al. [16] have reported that the frequency of the "crazy-paving sign" was 29\% and that of the "spider web sign" was $25 \%$. The reverse halo sign (atoll sign) (i.e., areas of GGO with peripheral consolidation) is frequently observed [3]; in this study, the

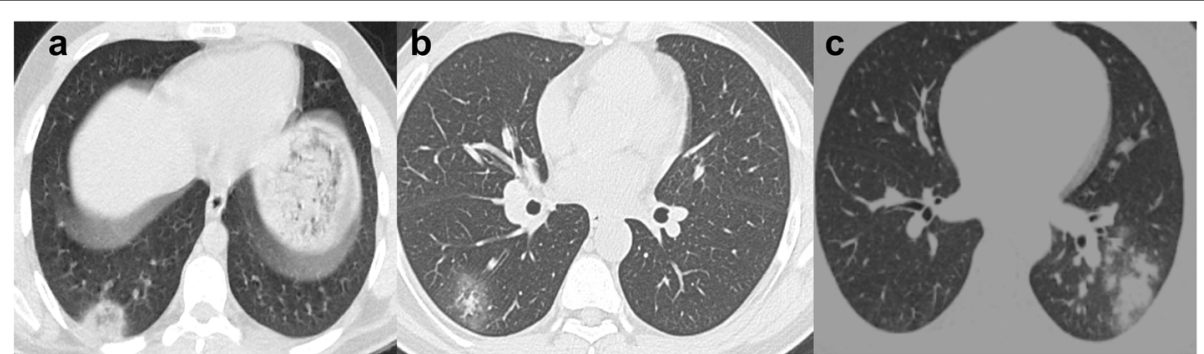

Fig. 4 Axial chest CT lung window in different cases with COVID-19 pneumonia showing a the reversed halo sign: right lower lobe posterior basal segment patchy consolidation with internal lucency. $\mathbf{b}$ Halo sign: patchy consolidation with a surrounding halo of ground-glass density at the right lower lobe apical segment (b) and at the left lower lobe (c) 


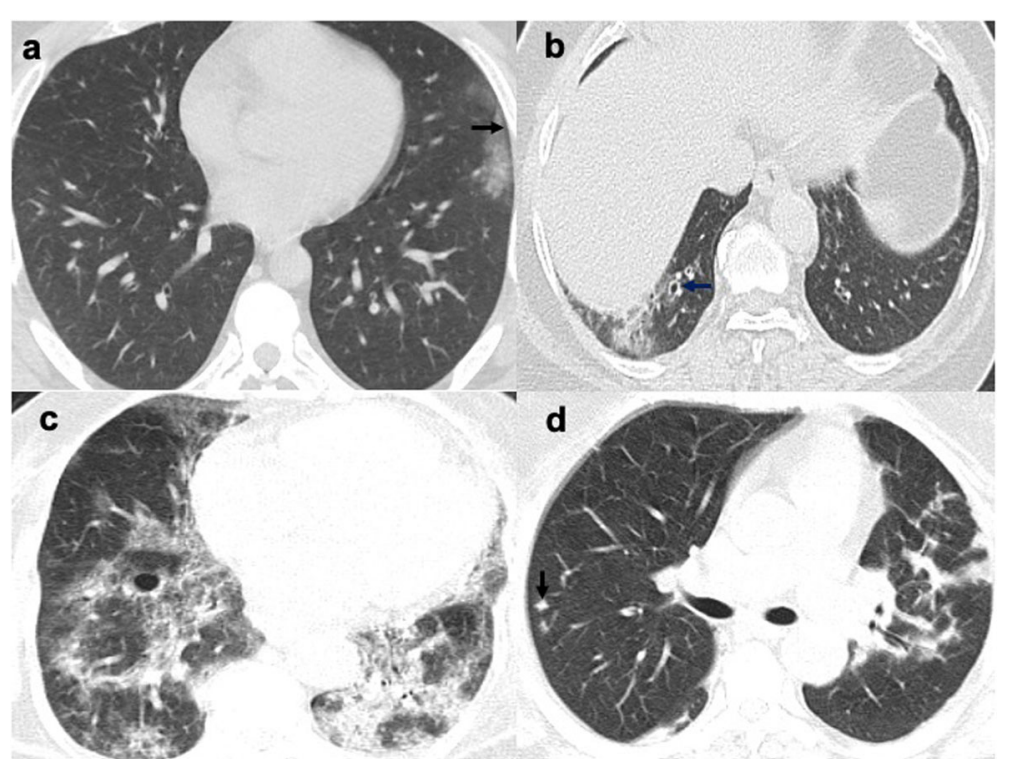

Fig. 5 Axial chest CT lung window in cases with COVID-19 pneumonia. a The patchy ground-glass density with subpleural lucent line (arrow). b Right patchy ground-glass opacity, bronchial dilatation, and thickening (arrow). $\mathbf{c}$ The bilateral ground-glass densities and consolidation, with a right lower lobe cyst and a right apical segment $L L$ angulated shape well-defined nodule (d)

frequency of the reverse halo sign was $12 \%$. Furthermore, the halo sign, which is a consolidative nodule or mass with peripheral GGO, was found in $11 \%$ of the patients in this study; it is uncommon in adults and could reach $50 \%$ in children [18]. The pleural transparent line or subpleural sparing is another CT sign found in $26.5 \%$, whereas in previous studies, the reported frequency of subpleural sparing was $6-53.2 \%[15,16]$.

In this study, bronchial changes (bronchial thickening and distortion) were observed in $59 \%$ of the patients. Zhou et al. [15] have stated that $72.6 \%$ of the patients had air bronchogram, and $17.7 \%$ had bronchus distortion; however, Wu et al. [16] have reported that $11 \%$ of the patients had bronchial wall thickening.

In this study, $10 \%$ of the patients had air-containing cysts, which could be due to pathological dilatation or due to resorption of consolidation; some authors have described it as a cavity, and others called it cystic changes and cavity or bubble sign; this conforms to Shi et al.'s [12] study that found cysts in $10 \%$ of the cases. Nodules are characterized to be small (less than $3 \mathrm{~cm}$ ) round, oval, or irregularly shaped and well or poorly defined opacity in the lung. It was reported in 3-13\% of the COVID-19 CT cases, and most nodules are multifocal and irregular and can have a halo sign [19]. In this study, nodules were encountered in $20 \%$ of the patients.

In terms of pleural changes, in this study, pleural thickening was observed in $76.5 \%$ of the patients; however, pleural reaction and effusion were found in 1.5\% and $3.5 \%$ of the patients, respectively. Zhou et al. [15] have shown that $48.4 \%$ of the patients had pleural thickening. However, pleural effusion was not significantly associated with COVID-19 pneumonia; its frequency ranged from 6 to $9 \%$ of the cases $[15,16,20]$.

In this study, lymphadenopathy had a significant correlation with disease severity $(p=0.088)$. Lymphadenopathy

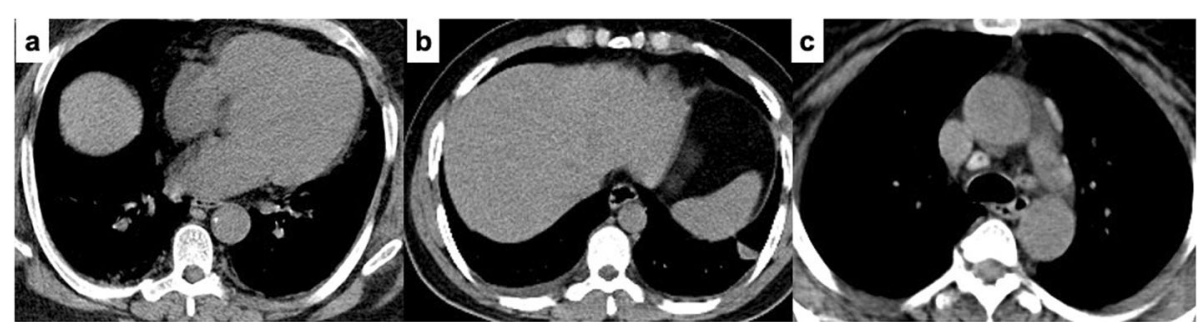

Fig. 6 Axial CT mediastinal window in different cases with COVID-19 pneumonia. a Bilateral pleural thickening. b Right mild size free pleural effusion and left pleural reaction. $\mathbf{d}$ Mediastinal reactive lymph nodes noted at the right paratracheal, aortopulmonary, and prevascular regions. Note the high density of lymph nodes 
Table 4 Number and percent of cases in each severity score group

\begin{tabular}{ll}
\hline CT severity score & Number of cases \\
\hline $1-5$ & $36(18 \%)$ \\
$6-10$ & $47(23.5 \%)$ \\
$11-15$ & $81(40.5 \%)$ \\
$16-20$ & $31(15.5 \%)$ \\
$21-25$ & $5(2.5 \%)$ \\
\hline
\end{tabular}

occurred predominantly in patients with a severe form of the disease [21]. Mediastinal lymph node enlargement was found in $43.51 \%$ of the patients with COVID-19 pneumonia with hilar, and mediastinal lymph node enlargement was associated with a 2.79-fold increased risk of COVID19 pneumonia [20]; however, Wu et al. [16] have reported that the frequency of mediastinal lymph node enlargement was $4 \%$ of the cases. In this study, lymphadenopathy was observed in $33 \%$ of the patients. The high density of lymph nodes without obvious calcification is not reported in the literature; therefore, it is suggested to be due to the association of CT evidence of old tuberculosis infection, which was noted in $12.5 \%$ of the cases.

The CT-SS of COVID-19 pneumonia has great significance in assessing the extent of pneumonia involvement, with differentiation of moderate, severe, and critical types, and in predicting the dynamic changes of chest CT follow-up exams in different severities of COVID-19 pneumonia. Furthermore, assessing the severity of COVID-19 in the early stage helps clinicians early and accurately treat the disease [22].

The CT-SS was proposed for the assessment of the extent of involvement in thin-section CT images. Zhou et al.'s scoring system divided both lungs into 12 zones altogether. The degree of involvement in each lung zone was scored from 0 to 4 , with a maximum possible score of 48 [15]. In another scoring system, both lungs were divided into 20 regions, evaluated on chest CT using a system attributing scores of 0,1 , and 2 ; therefore, the sum of the individual may range from 0 to 40 points [7]. In this study, the lobar involvement scoring system (025) was used as it was practical and time-saving with such a high flow of cases $[9,10]$.

Indicators of severe disease are marked tachypnea, hypoxemia, and infiltration of more than $50 \%$ of the lung fields [23]. According to Yang et al. [7], the optimal CTSS threshold for identifying severe COVID-19 was 19.5/ 40 , with $83.3 \%$ sensitivity and $94 \%$ specificity. In addition, Francone et al. [9] have stated that CT-SS of $\geq 18$ is highly predictive of patient mortality in short-term follow-up. Therefore, in this study, 18/24 was considered the cutoff value among the mild and severe cases. The mean global CT-SS was 11.2 in this study. In Francone et al.'s study [9], the mean global CT-SS was $12.3 \pm 11.1$.
The main limitations of this study are that the number of severe cases was much lesser than that of mild cases, which might affect the statistical strength, and single doctor assessment and the disease duration were unknown.

\section{Conclusion}

In Egyptian patients with COVID-19 pneumonia, multislice CT plays a vital role in early identification of the disease with the ground-glass densities, and peripheral adhesions were the most typical findings, Thus, radiologists should be familiar with other possible findings.

The assessment of the CT-SS of COVID-19 is essential in determining the extent of pneumonia involvement, to help clinicians early diagnosis and accurately treat COVID-19 pneumonia. The mean CT-SS for Egyptian patients with COVID-19 pneumonia was 11.2 (mild severity).

\section{Abbreviations}

COVID: Coronavirus disease; CT: Computed tomography; CT-SS: Computed tomography severity score; GGO: Ground-glass opacities; MSCT: Multi-slice computed tomography; SARS: Severe acute respiratory syndrome

\section{Acknowledgements}

I would like to thank the chest team for their case referral, participants who trusted us for their patience and support, and Dr. Mariam Hafez for her effort in the statistical analysis.

\section{Author's contributions}

$\mathrm{MH}$ developed the research idea, reviewed the literature, contributed to the data collection and analysis, organized the results, and wrote, revised, and approved the final manuscript.

\section{Funding}

This study had no funding from any resource.

\section{Availability of data and materials}

The datasets used and analyzed during the current study are available from the corresponding author on reasonable request.

\section{Ethics approval and consent to participate}

This study was approved by the Research Ethics Committee of the Faculty of Medicine at Cairo University in Egypt (number not available). All patients included in this study gave written informed consent to participate in this research.

\section{Consent for publication}

All patients included in this research gave written informed consent to publish the data contained within this study.

\section{Competing interests}

The author declares that there is no conflict of interest to declare.

Received: 7 October 2020 Accepted: 23 November 2020

Published online: 08 December 2020

\section{References}

1. Borghesi A, Zigliani A, Masciullo R, Golemi S, Maculotti P, Farina D, Maroldi D (2020). Radiographic severity index in COVID-19 pneumonia: relationship to age and sex in 783 Italian patients. La radiologia medica 1-4. https://doi. org/10.1007/s11547-020-01202-1.

2. Coronavirus Update (Live): 31,948,117 cases and 978,261 deaths from COVID-19 virus outbreak - Worldometer. https://www.worldometers.info/ coronavirus/. Accessed 23 Sept 2020.

3. Hani C, Trieua NH, Saaba I, Dangearda S, Bennani S, Chassagnona G, Revel MP (2020) COVID-19 pneumonia: a review of typical CT findings and differential diagnosis. Diagn Inter Imaging 101:263-268 
4. Corman VM, Landt O, Kaiser M et al (2020) Detection of 2019 novel coronavirus (2019-nCoV) by real-time RT-PCR. Euro Surveill 25(3):2000045

5. Wang $Y$, Dong C, Hu Y et al (2020) Temporal changes of $C$ f findings in 90 patients with COVID-19 pneumonia: a longitudinal study. Radiol 296(2):1-9

6. Li X, Zeng W, Chen $\mathrm{H}$ et al (2020) CT imaging changes of corona virus disease 2019 (COVID-19): a multi-center study in Southwest China. J Transl Med 18(154):1-8

7. Yang R, Li X, Liu H et al (2020) Chest CT severity score: an imaging tool for assessing severe COVID-19. Radiol Cardiothorac Imaging 2:2

8. COVID working group of the Dutch Radiological Society, 25-3-2020.

9. Francone M, lafrate F, Masci GM et al (2020) Chest CT score in COVID-19 patients: correlation with disease severity and short-term prognosis. Eur Radiol 4:1-10

10. Pan F, Ye T, Sun P, Gui S, Liang B, Li L, Zheng D, Wang J, Hesketh RL, Yang $L$, Zheng C (2020). Time Course of Lung Changes at Chest CT during Recovery from Coronavirus Disease 2019 (COVID-19). Radiology 295:715721.

11. Chung M, Bernheim A, Mei X et al (2020) CT imaging features of 2019 novel coronavirus (2019-nCoV). Radiol 295(1):202-207

12. Shi XH, Jiang N, Cao Y et al (2020) Radiological findings from 81 patients with COVID-19 pneumonia in Wuhan, China: a descriptive study. Lancet Infect Dis 20(4):P425-P434

13. Bai H, Hsieh B, Xiong Z et al (2020) Performance of radiologists in differentiating COVID 19 from viral pneumonia on chest CT. Radiol. Published Online:E46-E45

14. Salehi S, Abedi A, Balakrishnan S, Gholamrezanezhad A (2020) Coronavirus disease 2019 (COVID-19): a systematic review of imaging findings in 919 patients. AJR Am J Roentgenol;215: 87z93.

15. Zhou S, Wang Y, Zhu T, Xia L (2020) CT features of coronavirus disease 2019 (COVID-19) pneumonia in 62 patients in Wuhan, China. AJR 214:1-8

16. Wu J, Wu X, Zeng W et al (2020) Chest CT findings in patients with coronavirus disease 2019 and its relationship with clinical features. Invest Radiol 55(5):257-261

17. Liab M (2020) Chest CT features and their role in COVID-19. Radiol Infect Dis 2(7):51-54

18. Azadbakht J, Haghi-Aminjan H, Farhood B (2020) Chest CT findings of COVID-19-infected patients, are there differences between pediatric and adult patients? A systematic review. Egypt J Radiol Nucl Med 51:145

19. Ali TF, Tawab MA, ElHariri MA (2020). CT chest of COVID-19 patients: what should a radiologist know? Egypt I Radiol Nucl Med 51, 120. https://doi.org/ https://doi.org/10.1186/s43055-020-00245-8.

20. Li X, Fang X, Bian Y et al (2020) Comparison of chest CT findings between COVID-19 pneumonia and other types of viral pneumonia: a two-center retrospective study. Eur Radiol 30:5470-5478

21. Yu M, Xu D, Lan L, Tu M, Liao R, Cai S, Cao Y, Xu L, Liao M, Zhang X, Xiao S, Li Y, Xu H (2020) Thin-section chest CT imaging of coronavirus disease 2019 pneumonia: comparison between patients with mild and severe disease. Radiology Cardiothorac Imaging 2(2)

22. Liu N, He G, Yang X et al (2020) Dynamic changes of chest CT follow-up in coronavirus disease-19 (COVID-19) pneumonia: relationship to clinical typing. BMC Med Imaging 20:92

23. Gandhi RT, Lynch JB Carlos del Rio (2020) Mild or moderate Covid-19. N Engl J Med:1-9

\section{Publisher's Note}

Springer Nature remains neutral with regard to jurisdictional claims in published maps and institutional affiliations.

\section{Submit your manuscript to a SpringerOpen ${ }^{\circ}$ journal and benefit from:}

- Convenient online submission

- Rigorous peer review

- Open access: articles freely available online

High visibility within the field

- Retaining the copyright to your article

Submit your next manuscript at $\boldsymbol{\nabla}$ springeropen.com 